\title{
Interférences
}

Ars scribendi

$6 \mid 2012$

La question de la littérarité

\section{Littérarité et exégèse à Rome}

Quelques réflexions en marge d'une lettre de Sénèque

Jean-Christophe Jolivet

\section{CpenEdition}

Journals

Édition électronique

URL : http://journals.openedition.org/interferences/172

DOI : 10.4000/interferences. 172

ISSN : $1777-5485$

Éditeur

HiSoMA - Histoire et sources des Mondes antiques

Édition imprimée

Date de publication : 1 janvier 2012

ISSN : 1777-5485

Référence électronique

Jean-Christophe Jolivet, «Littérarité et exégèse à Rome », Interférences [En ligne], 6 | 2012, mis en

ligne le 11 décembre 2014, consulté le 15 septembre 2020. URL : http://journals.openedition.org/

interferences/172 ; DOI : https://doi.org/10.4000/interferences.172

Ce document a été généré automatiquement le 15 septembre 2020.

Tous droits réservés 


\title{
Littérarité et exégèse à Rome
}

\author{
Quelques réflexions en marge d'une lettre de Sénèque
}

\author{
Jean-Christophe Jolivet
}

La question de la littérarité semble s'être posée de façon multiple aux Anciens et particulièrement aux Romains: des textes illustres relevant apparemment de la paralittérature posent des cas d'école remarquables dans l'historiographie comme dans le domaine de la poésie ou de l'historia fabularis. Ainsi pourra-t-on invoquer le cas des commentarii de César, ouvrage exhibant une simplicité stylistique liée à la nature plus ou moins nettement administrative de l'œuvre, lui donnant l'apparence d'un compte rendu ${ }^{1}$; un autre cas remarquable, quoique moins illustre, est celui de l'hypomnèmation adressé au premier des élégiaques latins, Cornelius Gallus, par le poète hellénistique Parthénios de Nicée, que nous connaissons sous le nom d'Erotika pathèmata ${ }^{2}$. Dans les deux cas, ces textes font profession de fournir la matière brute pour des œuvres littéraires relevant d'un genre noble, de l'historia ornata dans le cas des commentaires de César, de l'élégie et de l'épopée dans le cas des Passions d'amour de Parthénios. On sait pourtant que ces textes, en dépit de leur profession d'humilité - explicite ou implicite et de leur annexion à un type d'écrits en principe infra-littéraire, présentent des caractéristiques qui relèvent pleinement de la littérarité et les font entrer de plein droit dans le champ de la littérature. La recherche des critères de la littérarité est une question qui se pose encore, dans les termes de l'époque, de façon cruciale dans l'Épitre à Auguste d'Horace. Le poète y dénonce le goût archaïsant de ses contemporains qui leur fait priser des textes d'origines diverses que les modernes excluraient sans l'ombre d'une hésitation du champ de la littérature.

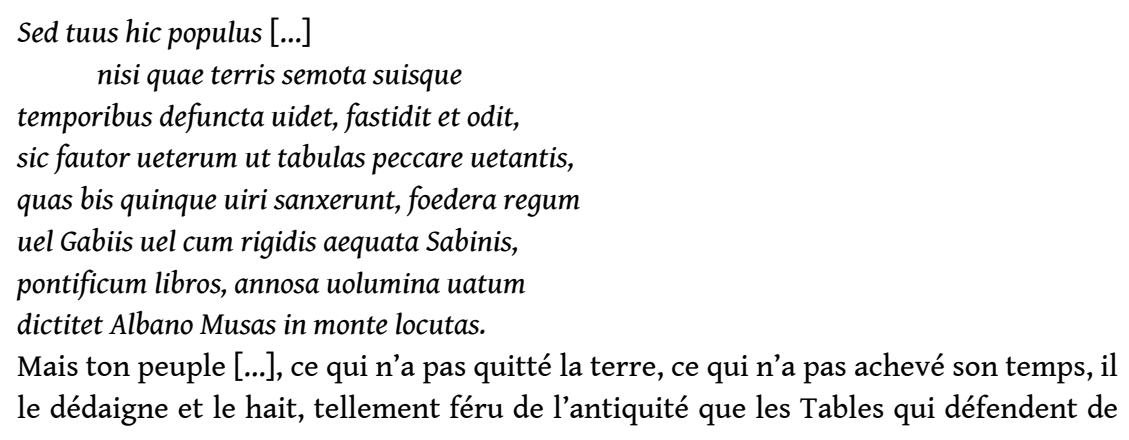


faillir, ces tables que des hommes au nombre de deux fois cinq ont établies, les traités conclus à conditions égales entre nos rois et Gabies ou les rigides Sabins, les livres des pontifes, les volumes chargés d'ans des devins, il va répétant que ce sont les Muses qui les ont prononcés sur le Mont Albain.

2 La dernière formule est un gage de la littérarité de ces textes aux yeux du public, à tout le moins de leur prestige dans le domaine des belles-lettres ${ }^{3}$. Or, si l'on en croit Horace, ces textes finissent par constituer, aux yeux de ses contemporains, une sorte de canon des textes archaïques: loi des Douze Tables, carmina Marciana, traités diplomatiques avec Gabies... L'archaïsme de ces vénérables reliques, que nous dirions non littéraires, constituait apparemment à lui seul un critère de littérarité pour les contemporains et il est bien difficile de déterminer s'il n'y a là que propos satirique de la part d'Horace. Disons-le d'emblée, il semble que ni de simples critères stylistiques, ni un contenu fictionnel ou au contraire scientifique et technique ne soient des critères suffisants de littérarité pour les Anciens.

3 Je voudrais poser la question du statut des textes exégétiques dans l'Antiquité. La question de leur littérarité, de leur appartenance à la littérature, n'a sans doute pas reçu une réponse uniforme au cours des siècles. L'opinion commune hésitera ainsi sans doute à classer d'emblée les textes critiques contemporains (Barthes, Blanchot, Genette et autres) dans la catégorie de la littérature alors qu'il ne viendrait sans doute à personne l'idée d'exclure les écrits exégétiques patristiques du champ de la littérature chrétienne (commentaires sur les psaumes, sur tel ou tel livre de l'Ancien Testament, etc.). Qu'en était-il de l'exégèse, et particulièrement de l'exégèse des poètes, plus largement de la littérature critique concernant la poésie, dans la Rome républicaine et impériale? Si l'on a recours à la catégorie genettienne du paratexte, il semble bien que celle-ci doive être résolument exclue du champ littéraire.

4 Les ouvrages qui relèvent de ce que l'on désigne parfois sous l'expression de PeriLiteratur, c'est-à-dire des monographies critiques sur tel problème grammatical, tel passage, telle question mythographique, notamment chez Homère, étaient légion dans l'Antiquité et les fonds des bibliothèques hellénistiques étaient sans doute composés essentiellement de ces traités ${ }^{4}$. Comme on le sait, la philologie latine a imité la philologie hellénistique et nous avons quelques témoignages de ce type pour la philologie virgilienne, voire prévirgilienne : certains érudits traitaient de quaestiones mythographiques complexes (peut-être antérieures à l'Énéide il est vrai), tel Ateius Philologus, dans un opuscule intitulé An amauerit Didun Aeneas ${ }^{5}$. Ce type de traités dérive sans doute de la tradition aristotélicienne des aporèmata homerica, genre qui a lui-même subsisté de façon autonome, par exemple avec les Questions homériques de Porphyre. Ces questions de tous ordres sur les poètes, la poésie ou l'historia fabularis ont bien sûr pu constituer l'objet d'un opuscule ou simplement d'un développement dans un ouvrage critique aux ambitions plus larges. Il n'en reste pas moins qu'il s'agit d'un type d'écrits bien identifié. Il se trouve que nous possédons au moins un texte latin qui nous donne des indications très nettes sur l'appartenance ou non de tous ces textes critiques à la littérature et nous propose par là même des critères de littérarité. Il s'agit d'une lettre de Sénèque dont l'objet essentiel est la critique de la paideia. Le philosophe s'en prend vivement aux érudits de son époque et particulièrement à Didyme, l'un des commentateurs alexandrins les plus prolixes, un champion de la Peri-Literatur et des questions philologiques ${ }^{6}$ :

Le grammairien Didyme a composé quatre mille volumes; je le plaindrais même s'il

n'avait fait que lire un tel ramassis d'inutilités. Dans tel groupe de traités, la 
question est de savoir quelle fut la patrie d'Homère; dans tel autre qui était véritablement la mère d'Énée; ailleurs si la vie d'Anacréon fut plutôt celle d'un débauché ou plutôt celle d'un ivrogne, si Sapho était une courtisane, sans compter d'autres fadaises, qu'il faudrait désapprendre si on les savait. Vraiment, je compulserai l'histoire de toutes les nations pour savoir qui le premier a fait des vers; je supputerai, sans fastes pour me documenter, le laps de temps qui sépare Orphée d'Homère; je passerai en revue les signes dont Aristarque embrochait les vers d'autrui et je consumerai mes jours sur des syllabes ${ }^{7}$ ?

Sénèque a évoqué plus haut une série de questions du même genre; il oppose à cette philologie bornée une réflexion sur l'utilité et la dignité de la lecture des grands classiques :

Quant à rechercher qui est l'aîné d'Homère ou d'Hésiode, ce point ne présente pas plus d'intérêt pour moi que la question de savoir pourquoi Hécube, cadette d'Hélène, portait si mal son âge. Dis-moi encore, je te prie, si rechercher l'âge de Patrocle et d'Achille te semble d'un intérêt capital. Tu te demandes en quelle contrée errait Ulysse au lieu de nous apprendre le moyen de ne pas errer toujours? Je n'ai pas le temps d'écouter quand on se demande devant moi s'il fut battu du flot et des orages entre l'Italie et la Sicile ou au-delà des limites du monde connu, vu qu'il lui était impossible d'errer si longtemps dans un trop étroit espace. Les tempêtes, celles de l'âme, nous secouent, nous autres, chaque jour ; nos mauvaises passions nous engagent dans toutes les mésaventures d'Ulysse. Nous rencontrerons la beauté, propre à solliciter nos yeux; et tant d'ennemis! Ici d'implacables monstres, friands de sang humain; là, d'insidieuses douceurs qui charment nos oreilles; là encore, des naufrages et toutes les variétés du malheur. Enseigne-moi comment je dois aimer ma patrie, ma femme, mon père; comment je dois, sur un débris de nef naufragée, courir à d'aussi nobles sujets. Pourquoi examines-tu si Pénélope n'aurait pas été impudique, si elle n'aurait pas mystifié son siècle, si elle ne soupçonnait pas, avant d'en être assurée, que celui qu'elle voyait était Ulysse? Enseigne-moi ce que c'est que la chasteté et tout le prix de cette vertu, si elle tient au corps ou à l'âme ${ }^{8}$.

La lettre 88 contient une critique en règle des arts libéraux et de la spécialisation de certaines disciplines, dont la grammatica, au nom de la supériorité de la philosophie, et prône une forme d'ascétisme culturel, dans une perspective de critique de l'enkuklios paideia, d'inspiration épicurienne et cynique ${ }^{9}$. Sans qu'il soit besoin de revenir sur les détails précieux qu'elle nous donne sur la production alexandrine en matière d'exégèse et d'histoire littéraire à la fin de la République et au début de l'Empire, nous pouvons constater qu'elle nous fournit des indications sur les critères selon lesquels ce type de textes peuvent être exclus des écrits dignes d'être lus : si les ouvrages de Didyme ou d'Aristarque ne sont pas dignes d'être lus, c'est qu'ils présentent un caractère de technicité bien trop élevé et s'occupent de questions ridicules. Les livres produits par la cupiditas sciendi ne relèvent pas de la littérature. À cette aune, l'érudition est un critère d'exclusion du champ du littéraire. Par ailleurs, le passage de Sénèque nous indique comment lire les grands textes épiques: il s'y trouve un enseignement moral, une allégorie éthique, et l'on peut y chercher des moyens d'amélioration personnelle. Voilà, en somme, ce qu'est la littérature. À partir de ces données, on pourra facilement considérer que, pour Sénèque, il est deux critères de littérarité essentiels : le caractère non technique d'un texte (l'excès de précision technique est critiqué dans cette lettre même en matière de philosophie) et d'autre part sa capacité à offrir un enseignement moral : en somme, la littérature se définit ici par sa capacité à être magistra uitae, pour paraphraser Cicéron. Voilà donc deux critères clairement donnés. 
7 Mais ces critères de détermination du champ de la véritable littérature restent bien évidemment parfaitement théoriques, inspirés par une perspective philosophique; ils ont une valeur normative quant au bon usage de la lecture et de la culture en général et c'est à ce titre qu'ils consacrent la condamnation d'une certaine conception de la paideia ; cela n'empêche pas la Peri-Literatur de rencontrer un succès inattendu auprès du public lettré, succès qui lui confère une dignité remarquable et un étrange pouvoir d'attraction en dépit de sa technicité et de son haut degré de spécialisation. De tels ouvrages jouent, à l'occasion au moins, et malgré leur caractère pointu, le même rôle que des textes plus prestigieux dans les pratiques de l'amicitia littéraire. Du point de vue d'une sociologie de la lecture, ils ont une fonction analogue à celle des grandes œuvres littéraires. C'est le cas d'un traité de prosodie homérique du grammairien Tyrannion d'Amissos que Cicéron reproche à Atticus d'avoir fait lire en son absence, manquant aux officia de l'amitié littéraire. Faute d'avoir pu assister à cette première, Cicéron demande donc à Atticus de lui faire parvenir l'ouvrage, tout en faisant mine, avec une hauteur toute philosophique, de trouver un tel sujet trop pointu ${ }^{10}$ :

Venio ad Tyrannionem. Ain tu? uerum hoc fuit? sine me? At ego quotiens, cum essem otiosus, sine te tamen nolui? Quo modo hoc ergo lues? Vno scilicet si mihi librum miseris; quod ut facias etiam atque etiam rogo. Etsi me non magis liber ipse delectabit quam tua admiratio delectauit. Amo enim panta philêdeimona teque istam tam tenuem theôrian tam ualde admiratum esse gaude, etsi tua quidem sunt eius modi omnia. Scire enim uis; quo uno animus alitur. Sed, quaeso, quid ex ista acuta et graui refertur ad telos ? [...] Librum, si me amas, mitte. Tuus est enim profecto, quoniam quidem est missus ad te.

J'en viens à Tyrannion : dis-moi, le procédé était-il vraiment correct? faire cela sans moi ? Moi, au contraire, combien de fois, étant de loisir, je me suis néanmoins abstenu, parce que tu n'étais pas là? Comment résoudras-tu cette difficulté ? Un seul moyen, évidemment: m'envoyer le livre; je te le demande instamment. D'ailleurs le livre lui-même ne me procurera pas plus de plaisir que ne m'en a procuré ton admiration pour lui. J'aime en effet en toi l'amateur universel et je me réjouis que tu aies si fort admiré une étude si subtile. Mais chez toi, tout est de cette veine : tu veux savoir; de fait, le savoir est la seule nourriture de l'âme. Mais, s'il te plaît, quel rapport peuvent avoir ces «aigus » et ces "graves" avec le souverain bien ? [...] Si tu m'aimes, envoie-moi le livre. Car il t'appartient sûrement, puisqu'il t'a été envoyé ${ }^{11}$.

8 Ce qu'il y a d'intéressant dans la lettre de Cicéron, c'est qu'elle fait allusion à une position analogue à celle de Sénèque : les textes critiques ne sont pas propices à une recherche de caractère éthique. Toutefois, le fait que la lecture d'un tel ouvrage puisse faire l'objet d'un reproche pour manquement aux convenances des pratiques de l' amicitia lettrée prouve que des textes de ce type n'étaient pas, de ce point de vue, considérés comme fondamentalement différents de textes plus évidemment littéraires. Une telle lecture relève bien des activités lettrées de l'otium cum studio (là encore sans doute un critère important de littérarité) et cela suggère - mais le dire revient à enfoncer une porte ouverte - que la frontière entre textes techniques et scientifiques et textes littéraires était beaucoup moins clairement marquée pour les Anciens que pour nous.

9 La paralittérature exégétique remporte en fait un succès que l'on mesure mal car elle devient rapidement, dans le monde hellénistique puis à Rome, une sorte d' Unterhaltungsliteratur, littérature de pur divertissement, dans la mesure où elle met en œuvre des jeux lettrés, sans doute par imitation de ce qui se passait dans les cours hellénistiques d'Alexandrie ou de Pergame. 
10 C'est particulièrement le cas de la littérature de problèmata ou de quaestiones homériques ou virgiliens, dénoncée par Sénèque : quittant le champ de la littérature spécialisée, ceux-ci s'illustrent, dès avant l'époque impériale, dans la vie littéraire à Rome. Cet engouement en vient même à devenir une véritable maladie, si l'on en croit le De breuitate uitae (13), où Sénèque le vilipende en ces termes :

Morbus fuit quaerere quem numerum remigum Vlysses habuisset, prior esset scripta Ilias an Odyssea, praeterea an eiusdem esset auctoris.

Ce fut une maladie que de rechercher quel était le nombre des rameurs d'Ulysse, si c'était l'Iliade ou l'Odyssée qui avait été composée en premier lieu et en outre si elles étaient du même auteur.

11 Nous avons quelques témoignages de l'intrusion de ces questions exégétiques dans la vie scolaire. Juvénal campe un parent d'élève qui teste la connaissance de Virgile d'un grammaticus en lui demandant qui était la nourrice d'Anchise, le nom et la patrie de la nouerca d'Anchemolus ${ }^{12}$, le nombre d'années qu'Aceste vécut ${ }^{13}$, le nombre d'amphores de vin données par les Siciliens aux Troyens ${ }^{14}$, questions analogues à celles dont Sénèque raillait la vanité ${ }^{15}$, mais qui manifestent - fût-ce sur un ton satirique l'importance de la littérature exégétique virgilienne dans l'enseignement scolaire ${ }^{16}$. Ce genre de sport cérébral est aussi pratiqué à la cour des Césars. Le modèle de ce type d'entretien princier lettré remonte à Alexandre lui-même. Les empereurs romains pratiquèrent tout naturellement en ce domaine l'imitatio Alexandri: Ptolémée Héphestion met ainsi en scène une question homérique traitée à la table d'Auguste ${ }^{17}$, Tibère interroge les philologues de sa cour : sur la mère d'Hécube, le nom qu'Achille portait à Scyros parmi les filles de Lycomède ${ }^{18}$, ou ce que chantaient les sirènes ${ }^{19}$. Cette Unterhaltungsliteratur fait donc florès; il est de bon ton d'imiter les pratiques littéraires des grands centres culturels et des sociétés hellénistiques.

12 Toutefois, l'avènement de ces questionnements philologiques et exégétiques à la littérature s'effectue de façon manifeste, si l'on adopte un point de vue moderne, le jour où ils entrent en fiction et accèdent au statut d'argumentum d'œuvres fictives, avec Plutarque, Aulu-Gelle, Athénée, Macrobe, et d'autres : non contentes d'envahir la vie lettrée, à l'école, à la cour, dans les banquets, les questions exégétiques et poétiques deviennent le sujet même des œuvres de fiction.

13 L'effort de Sénèque pour exclure cette paralittérature du champ des digna se trouve ainsi battu en brèche par l'émergence d'une littérature de propos de table. La pratique grammaticale et scolaire de l'explicatio quaestionum contribue à l'émergence d'un genre symposiaque bien identifié : questions grecques et romaines de Plutarque, Nuits attiques d'Aulu-Gelle, Deipnosophistes d'Athénée..., où toutes les formes d'érudition trouvent leur place, parmi lesquelles l'exégèse et la philologie.

14 Ce qui est le plus technique, le moins digne d'être lu, si l'on retient les critères de Sénèque, devient ainsi objet de littérature, et c'est même la virtuosité exégétique des philologues qui devient matière à fiction : l'un des meilleurs exemples en est la mise en scène par Plutarque du problème de savoir à quelle main Diomède blesse Aphrodite dans l'Iliade. L'élaboration virtuose de la lysis est exposée par le menu dans les Propos de table $(9,4)$ par le rhéteur Maximus ${ }^{20}$. Les héros de l'épopée sont remplacés par ceux de l'érudition. Athénée met en scène un banquet où l'amphitryon romain Publius Livius Larensis fait preuve de virtuosité en matière d'homeric scholarship, et reçoit le surnom d' Asteropaeus car, de même que le héros homérique décochait indifféremment ses traits de la main droite ou de la gauche (Il.21, 163-164), de même Larensis citait aussi 
aisément en latin et en grec et soumettait ses convives à de difficiles exercices de citation homérique ${ }^{21}$. Trimalcion, on s'en doute, n'eût pas brillé à un tel banquet ${ }^{22}$. Ainsi l'on assiste à une fictionnalisation de l'exégèse et de la Peri-Literatur qu'elle engendre. Les auteurs mettent en scène la pratique symposiaque de la résolution de problèmes et l'exégèse devient pleinement littérature.

Mais il y a plus grave : cette paralittérature, non contente de constituer le sujet même des textes symposiaques, pousse l'audace jusqu'à s'insinuer dans l'épopée et dans les textes les plus prestigieux, les plus canoniques, au cœur de la fabula. On ne s'étonnera certes pas que ce soit le cas chez Ovide dont les Métamorphoses constituent une sorte d'anthologie des genres littéraires de l'Antiquité : même la littérature de quaestiones y a droit de cité. Lors du banquet qui suit les noces de Persée et d'Andromède, au chant 4 des Métamorphoses, Persée a narré de façon expéditive sa geste héroïque. Ce récit est motivé par des quaestiones convivales dont la formulation même évoque inévitablement une approche mythographique :

Fare, precor, Perseu, quanta uirtute quibusque

Artibus abstuleris crinita draconibus ora.

Dis-nous, s'il te plaît, ô Persée, par quelle vaillance et quels artifices tu t'es emparé

de la tête aux cheveux de serpents ${ }^{23}$.

Persée évoque alors sa geste en une espèce de catalogue au style indirect, au risque (ou avec l'intention délibérée) de décevoir l'attente de son auditoire (intra- et extradiégétique: ante exspectatum tacuit ${ }^{24}$ ). Vient se substituer au récit épique - claire allusion, là encore, à la pratique lettrée symposiaque - une question d'érudition mythologique relative aux cheveux de Méduse ; elle se trouve plaisamment valorisée au détriment du récit héroïque, en un étonnant retournement des hiérarchies génériques traditionnelles, par une remarque du héros-narrateur :

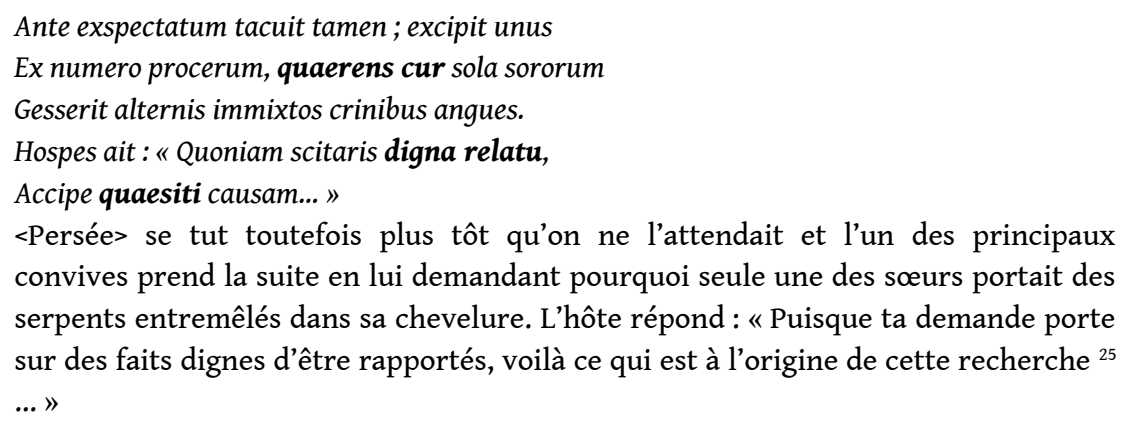

17 Le vieux récit épique cède ainsi la place à la moderne Unterhaltungsliteratur des banquets, faite de zètèmata mythographiques et d'étiologies. La quaestio, ici valorisée comme digna relatu, a donc supplanté l'épopée. En somme, Persée adopte la position inverse de celle de Sénèque et se fait le défenseur d'une forme d'érudition un peu vaine qui caractérise l'enkuklios paideia. Passe encore que ce provocateur d'Ovide se permette de telles irrévérences par rapport aux hiérarchies établies; mais un phénomène analogue s'observe jusque dans le texte littéraire latin par excellence, le premier poème à Rome, le deuxième dans le monde antique : l'Énéide de Virgile. L'exemple le plus éclatant de cette projection des quaestiones homericae dans l'historia fabularis se situe lors du banquet de Carthage ; Didon pose en effet au héros un certain nombre de questions sur la guerre de Troie :

Multa super Priamo rogitans, super Hectore multa;

nunc quibus Aurorae uenisset filius armis,

nunc quales Diomedis equi, nunc quantus Achilles. 
Posant de nombreuses questions sur Priam, sur Hector, demandant tantôt avec quelles armes était venu le fils de l'Aurore, tantôt comment étaient les chevaux de Diomède, tantôt quelle était la taille d'Achille ${ }^{26} .$. homériques ou post-homériques.

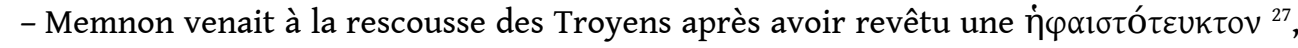
une panoplie fabriquée par Héphaïstos, comme celle d'Achille, comme la cuirasse de Diomède $(I l .8,195)$ et, bientôt, comme les armes d'Énée. Or, c'est bien en raison de ce caractère divin des armes, précise Servius, que Didon pose la question (ad Aen. 1, 751): ideo autem dixit quibus armis, quia Vulcaniis armis usus fuisse narratur, «<Virgile> a dit: avec "quelles armes" précisément parce que, selon la tradition, <Memnon> est venu avec des armes faites par Vulcain "; il y avait sans doute là de quoi se livrer aux délices de l'exégèse allégorique tout au long d'une nuit carthaginoise, sur le modèle de l'exégèse du bouclier d'Achille.

- La taille d'Achille était une question abordée notamment dans le cadre de zètèmata concernant la nécessité, pour le héros, de se procurer une nouvelle panoplie après qu'il a perdu la sienne, prêtée à Patrocle (il ne revêt pas les armes de Patrocle au chant 19, et précise que seul le bouclier d'Ajax pourrait, à la rigueur, lui convenir) ${ }^{28}$.

- L'identification des chevaux de Diomède posait une difficulté, suite à la capture par le héros homérique des chevaux de Rhésos, lors de la Dolonie ; leur pedigree, le fait qu'ils descendent des cavales anthropophages du roi thrace Diomède, prises par Hercule, était l'occasion de savantes discussions, comme l'indique la note de Servius à la question de Didon ${ }^{29}$.

Il y a donc là, à l'évidence, un échantillonnage de questions classiques, avec une tendance, peut-être, à privilégier une mythographie de mirabilia et des questions allégoriques - on ne saurait toutefois s'étonner plus que de mesure que Didon ne pose pas à Énée des questions aristarchéennes de prosodie homérique comme le font Cicéron et Atticus. La scène virgilienne procure ainsi une archéologie de la pratique symposiaque et des zètèmata hellénistiques en représentant une espèce d'Urphilologie à l'œuvre dans le monde hérö̈que. Celle-ci constituait donc en somme l'une des premières formes de vie littéraire!

L'on en arrive donc à projeter les quaestiones convivales comme Unterhaltungsliteratur dans le monde de l'épopée. Sénèque aurait sans doute été bien contrarié de voir que, selon certains lecteurs, c'est même dans le premier texte du monde gréco-latin, chez Homère, que les superuacua de Didyme trouvent leur origine. Ainsi, à en croire Athénée :

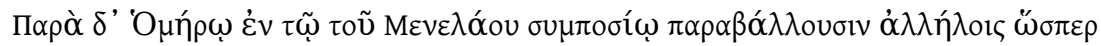
$\dot{\varepsilon} v \delta 1 \alpha \tau \rho \imath \beta \tilde{n} \zeta \eta \tau \eta \dot{\mu} \mu \alpha \tau \alpha$.

Chez Homère, lors du banquet de Ménélas, <les convives> se proposent les uns aux autres des questions, comme par passe-temps ${ }^{30}$.

La richesse de Ménélas provoque l'étonnement de Télémaque :

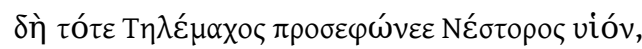

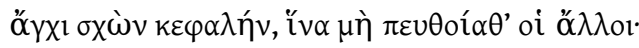

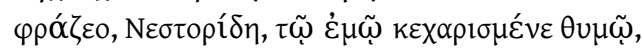

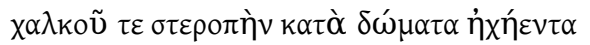

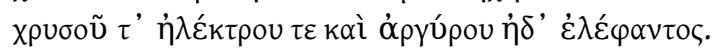

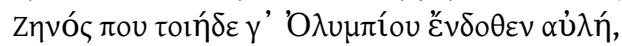

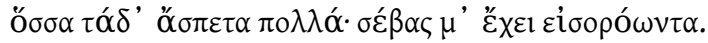

Interférences, 6 | 2012 
Télémaque, s'adressant au fils de Nestor, la tête toute proche, de peur que les autres ne fussent informés, lui dit : «Remarque, fils de Nestor cher à mon cœur, l'éclair du bronze par la demeure retentissante, celui de l'or, de l'électre, de l'argent, de l'ivoire. Tel est sans doute l'intérieur du palais de Zeus Olympien. Combien de choses ici vous laissent sans voix! Quand je les regarde, j'ai l'impression d'un miracle ${ }^{31}$.» l'occasion de résoudre la question de la vaisselle de Ménélas et de sa richesse ${ }^{32}$, à partir de l'étonnement manifesté par Télémaque devant l'opulence de son hôte. Tout se passe comme si la question de Télémaque relayait au sein même du texte un zètèma dont la teneur pourrait être en substance : comment se fait-il que le palais de Ménélas soit si riche? La lysis est apportée par le maître des lieux qui évoque son enrichissement lors de son nostos. Le banquet de l'odyssée serait donc la première occasion de questions homériques. De telles scènes constituent en quelque sorte le prototype des pratiques symposiaques des savants homérologues et mythographes de l'époque impériale.

On le voit donc à travers les diverses fortunes de ces problèmata, la question de la littérarité des textes techniques, et particulièrement des commentaires littéraires antiques, est loin d'être simple à résoudre. Les cas que l'on vient d'envisager suggèrent que la littérarité, pour les Anciens, ne saurait se mesurer uniquement à partir de considérations stylistiques, génériques, ou en fonction de l'établissement de canons, même si ces éléments sont primordiaux ; les pratiques lettrées et livresques semblent essentielles et l'on pourrait peut-être suggérer, à la lumière des quelques exemples de pratiques romaines et de textes latins qui ont été proposés, un critère extérieur : les textes latins, du moins, voient leur littérarité consacrée quand les Romains mettent en pratique à leur endroit l'imitatio des pratiques lettrées hellénistiques. Ainsi, un critère essentiel de littérarité à Rome pourrait être l'imitatio ou l'aemulatio des pratiques culturelles, livresques, philologiques du monde hellénistique ${ }^{33}$.

\section{BIBLIOGRAPHIE}

BRINK C.O. 1982, Horace on Poetry. III, Epistles, Book II: The Letters to Augustus and Florus, Oxford.

CANFORA L. 1996, « Les bibliothèques anciennes et l'histoire des textes », in M. Baratin,

C. Jacob (éds), Le pouvoir des bibliothèques : la mémoire des livres en Occident, Bibliothèque Albin Michel de l'histoire, Paris, p. 261-272.

HAAS W. (éd.) 1977, Die Fragmente der Grammatiker Tyrannion und Diokles, Sammlung griechischer und lateinischer Grammatiker 3, Berlin - New York.

носк R.F. 2001, « Homer in Greco-Roman education », in D.R. McDonald (éd.), Mimesis and Intertextuality in Antiquity and Christianity, Studies in Antiquity and Christianity, Harrisburg (Pa.), p. 56-77.

JOLIVET J.-C. 2010, « L'apport des correspondances de Cicéron et de Sénèque à l'histoire de la philologie grecque à Rome ", in J. Desmulliez, C. Hoët-Van Cauwenberghe, J.-C. Jolivet (éds), L' étude des correspondances dans le monde romain de l'Antiquité classique à l'Antiquité tardive :

Interférences, 6 | 2012 
permanences et mutations, Actes $\mathrm{du} X X X I^{e}$ colloque international de Lille, 20-22 novembre 2008, TravauX et recherches, Villeneuve d'Ascq, p. 293-306.

LEDENTU M. 2004, Studium scribendi : recherches sur le statut de l'écrivain et de l'écriture à Rome à la fin de la République, Bibliothèque d'études classiques 39 , Louvain.

LiGHTFоOT J. (éd.) 1999, Parthenius of Nicaea. The Poetical Fragments and the Erotica pathemata, Oxford.

PFEIFFER R. 1968, History of Classical Scholarship from the Beginnings to the End of the Hellenistic Age, Oxford.

SCHWARTZ J. 1960, «Quelques quaestiones homericae et vergilianae chez les écrivains latins », in Hommages à Léon Herrmann, Latomus 44, Bruxelles, p. 698-701.

SHACKLETON BAILEY D.R. (éd.) 1980, Cicero's Letters to Atticus. V, 48-45 B.C. 211-354 (Books XI to XIII), Cambridge.

STÜCKELBERGER A. 1965, Senecas 88. Brief: über Wert und Unwert der freien Künste: Text, Übersetzung, Kommentar, Bibliothek der klassischen Altertumswissenschaften, Heidelberg.

VACHER M.-C. (éd.) 1993, Suétone. Grammairiens et rhéteurs, CUF. Série latine 310, Paris.

\section{NOTES}

1. Ledentu 2004, p. 214, 218-219.

2. Parth., praef. Sur ce texte, voir Lightfoot (éd.) 1999.

3. Brink 1982, p. 66.

4. Canfora 1996, p. 266, pour la bibliothèque d'Alexandrie.

5. Sur Ateius Philologus, né avant 86 av. J.-C., voir Suet., Gram.10, cf. aussi les notes de Vacher (éd.) 1993. L'ouvrage en question est cité par Charisius, GLK, 1, 127, 17.

6. Pfeiffer 1968, p. 216-217, 275-276. Cf. Jolivet 2010 pour une analyse des différentes questions évoquées ici.

7. Sen., Ep. 88, 38-39, trad. Veyne.

8. Sen., Ep. 88, 6-8, trad. Veyne.

9. Stückelberger 1965.

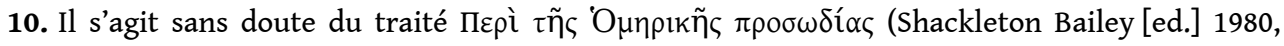
p. 306), que l'on connaît grâce aux scolies à Homère.

11. Cic., Att. 12, 6, 2 = Tyran. $\mathrm{T} 16$ Haas, trad. Beaujeu modifiée.

12. Verg., A. 10, 389.

13. Verg., A. 5, 73.

14. Verg., A. 1, 195.

15. Quint., Inst. 1, 2, 14, présente le fait d'expliquer les questions (quaestiones explicare) comme l'une des tâches du grammaticus.

16. Schwartz 1960.

17. Phot., Bibl. 151 a, à propos de Il. 4, 127.

18. Voir Ptolémée Chennos cité par Phot., Bibl. 190, p. 147 a 18.

19. Suet., Tib. $56 ; 70$.

20. Plut., Moralia 739 b-d.

21. Ath., Deipn. 14, 620 b.

22. Cf. Hock 2001, p. 69.

23. Ov., Met. 4, 770-771. 
24. Ov., Met. 4, 790.

25. Ov., Met. 4, 790-794.

26. Verg., A. 1, 750-752.

27. Procl., Chr. 172 Severyns.

28. Scolie bT ad Il. 16, 141-142.

29. Serv., A. 1, 752 : Quales Diomedes equi : [...] de his interrogat quos sustulit Rheso. « quales » autem dixit ac si diceret, anne sic feroces ut illi a quibus ducebant originem. Diomedes enim, rex Thracum, habuit equos qui humanis carnibus uescebantur. Hos Hercules occiso crudeli tyranno abduxisse perhibetur: de quibus dicuntur supra memorati equi originem ducere.

30. Ath., Deipn. 5, $188 \mathrm{~d}$.

31. Hom., Od. 4, 70-76.

32. Ath., Deipn. 5, 188 f - 189 a ; 190 a.

33. Hock 2001, p. 68.

\section{RÉSUMÉS}

À partir du constat que la définition de la littérarité et de ses critères s'est posée aux Anciens en termes variables et finalement assez flous pour nous, l'article envisage la question du statut littéraire ou non des textes exégétiques dans l'Antiquité pour montrer que la littérarité des Romains ne saurait se limiter à des critères stylistiques ou génériques traduits dans des canons esthétiques : la littérarité romaine passe également par le critère externe de l'imitatio et de l' aemulatio qui font entrer les textes nouveaux dans une tradition consacrée et les insèrent ainsi dans une littérarité de tradition.

\section{INDEX}

Mots-clés : littérarité, tradition interprétative, exégèse des poètes, philologie, littérature technique antique, morale et littérature, littérature symposiaque, étiologie, exégèse allégorique, quaestiones homericae, textes critiques

Keywords : literariness, interpretative tradition, poetic exegesis, philology, ancient technical literature, ethics and literature, allegorical interpretation, quaestiones homericae, critical texts, symposiac literature, etiology

nomsmotscles Aristarque de Samothrace, Atéius Philologus, Athénée, Aulu-Gelle, César, Cicéron, Didyme, Gallus, Homère, Horace, Macrobe, Ovide, Parthénios de Nicée, Photius, Plutarque, Porphyre, Ptolémée Héphestion, Sénèque, Suétone, Tyrannion d'Amissos, Virgile

\section{AUTEURS}

\section{JEAN-CHRISTOPHE JOLIVET}

Professeur de langue et de littérature latines

UMR 8164 HALMA-IPEL (CNRS - Lille 3 - MCC) 